\title{
Zebrafish mutants provide insights into apolipoprotein B functions during embryonic development and pathological conditions
}

\author{
Hanoch Templehof, Noga Moshe, Inbal Avraham-Davidi, and Karina Yaniv \\ Department of Biological Regulation, Weizmann Institute of Science, Rehovot, Israel.
}

\begin{abstract}
Apolipoprotein B (ApoB) is the primary protein of chylomicrons, VLDLs, and LDLs and is essential for their production. Defects in ApoB synthesis and secretion result in several human diseases, including abetalipoproteinemia and familial hypobetalipoproteinemia (FHBL1). In addition, ApoB-related dyslipidemia is linked to nonalcoholic fatty liver disease (NAFLD), a silent pandemic affecting billions globally. Due to the crucial role of $A P O B$ in supplying nutrients to the developing embryo, $A p o B$ deletion in mammals is embryonic lethal. Thus, a clear understanding of the roles of this protein during development is lacking. Here, we established zebrafish mutants for 2 apoB genes: $a p o B a$ and $a p o B b .1$. Double-mutant embryos displayed hepatic steatosis, a common hallmark of FHBL1 and NAFLD, as well as abnormal liver laterality, decreased numbers of goblet cells in the gut, and impaired angiogenesis. We further used these mutants to identify the domains within ApoB responsible for its functions. By assessing the ability of different truncated forms of human APOB to rescue the mutant phenotypes, we demonstrate the benefits of this model for prospective therapeutic screens. Overall, these zebrafish models uncover what are likely previously undescribed functions of $A$ poB in organ development and morphogenesis and shed light on the mechanisms underlying hypolipidemia-related diseases.
\end{abstract}

Conflict of interest: The authors have declared that no conflict of interest exists.

Copyright: @ 2021, Templehof et al. This is an open access article published under the terms of the Creative Commons Attribution 4.0 International License.

Submitted: May 20, 2019

Accepted: June 2, 2021

Published: July 8, 202

Reference information: /CI Insight. 2021;6(13):e130399.

https://doi.org/10.1172/jici. insight.130399.

\section{Introduction}

Apolipoprotein B (ApoB) is the primary structural component of atherogenic lipoproteins, such as chylomicrons, VLDLs, and LDLs, and is essential for their assembly (1). Elevated levels of LDL are widely recognized as a cardiovascular risk factor, and abundant data point to chemically modified LDL and ApoB as triggers of most of the features of the pathobiology of atherosclerosis. Besides the important role of APOB in lipid metabolism, a large body of data, accumulated during the past years, has revealed new roles for lipoproteins as signaling mediators in various cell types, operating at different levels and through various classic and nonclassic mechanisms (2).

Two APOB isoforms, APOB100 and APOB48, are present in humans and mice, which are encoded by a single gene and generated by RNA editing (3). Defects in ApoB synthesis and secretion, resulting from mutations in the $A p o B$, microsomal triglyceride transfer protein $(M T P)$, and proprotein convertase subtilisin/kexin type 9 genes, lead to abetalipoproteinemia (ABL) and familial hypobetalipoproteinemia (FHBL; refs. 4, 5), two disorders characterized by low or absent levels of ApoB and LDL-cholesterol in plasma. ABL and homozygous FHBL display similar clinical symptoms, including steatorrhea, neurological dysfunction, ophthalmologic abnormalities, and fatty liver. Since both disorders also carry a reduced risk of cardiovascular diseases (CVDs), research into the underlying molecular mechanisms could uncover attractive targets for lipid-lowering therapies in patients with hypercholesterolemia.

In addition to the involvement of $\mathrm{ApoB}$ in atherosclerosis and CVD, ApoB-related dyslipidemia is linked to another global epidemic, nonalcoholic fatty liver disease (NAFLD), which affects around $20 \%-30 \%$ of the adult population in Western countries (6). NAFLD results in steatosis, the accumulation of lipid in hepatocytes (7), which can lead to liver malfunction, cirrhosis, and, in some cases, hepatocellular carcinoma (8). Defects in production and/or secretion of ApoB lipoproteins are highly linked to hepatic steatosis (9). In particular, the impaired synthesis of VLDL observed in patients with FHBL, which results in triglyceride accumulation in the liver, represents one among many causes of NAFLD in humans (10). The crucial role 
ApoB plays in various pathological conditions has prompted the generation of several genetically modified mouse models $(11,12)$. However, because APOB is expressed in the yolk sac during early embryonic development, where it facilitates the supply of nutrients to the developing embryo, ApoB-knockout mice die at gestational stage 9.5 to 10.5 , thereby precluding the study of the roles of this protein during embryogenesis.

The close relationship between zebrafish and human apolipoprotein expression and function $(13,14)$ makes the former an attractive animal model for studying the repertoire of ApoB functions during embryonic development. In addition, the well-documented ability of zebrafish to develop hyperlipidemia $(15,16)$ and lipoprotein oxidation $(17,18)$ motivated the study of the cellular and molecular mechanisms linking lipoproteins and CVD in this animal. Here, we established mutants for 2 zebrafish apoB genes: $a p o B a$ and apoBb.1. We found that double-mutant embryos displayed lipid accumulation in hepatocytes, a common hallmark of FHBL1 and NAFLD. In addition, we detected early developmental defects, including abnormal liver laterality, decreased numbers of goblet cells in the gut, and impaired angiogenesis in double apoB mutants. Interestingly, we found altered Notch signaling to underlie some of the observed phenotypes. To identify the domains within ApoB associated with the antiangiogenic function, we assessed the ability of truncated forms of the human APOB (APOB25 and APOB34) to rescue the zebrafish mutant phenotypes. We found that only mutant embryos injected with APOB34 displayed a significant rescue of the vascular phenotypes, suggesting that the antiangiogenic activity of ApoB is contained between the 25 to $34 \mathrm{~N}$-terminus remnant of the $A$ poB protein. Altogether, these results uncover previously unappreciated functions of ApoB during early organ development and highlight the potential of these newly established apoB zebrafish mutants as models for studying human pathologies associated with hypolipidemia, as well as for related drug screens.

\section{Results}

Generation of zebrafish apoB mutants. Three $a p o B$ genes are detected in the zebrafish genome: apoBa, apoBb.1, and $a p o B .2(14,19)$. Two days postfertilization (dpf) embryos displayed strong expression of apoBa and apoBb.1 mRNA in the yolk syncytial layer (YSL), whereas apoBb.2 expression was undetectable (Figure 1, A, B, and E). At $5 \mathrm{dpf}, a p o B a$ expression became restricted to the liver (Figure 1C, arrowhead), and apoB. 1 was detected mostly in the YSL and the intestine (Figure 1D). To investigate the role of ApoB during embryonic development, we generated zebrafish carrying mutations in the $a p o B a$ and $a p o B b .1$ genes. sgRNAs targeting exon 5 of the $a p o B b .1$ gene (Supplemental Figure 1A; supplemental material available online with this article; https:// doi.org/10.1172/jci.insight.130399DS1) were injected along with cas9 mRNA into 1-cell-stage Tg(fli1:EGFP) embryos (20). F0 injected fish were raised to adulthood and screened for germline transmission. We identified 2 different mutations, including an $18 \mathrm{bp}$ insertion containing a premature in-frame stop-codon (Supplemental Figure 1A), which was used throughout this study. To target the apoBa gene, we used the transcription activator-like effector nuclease (TALEN) technology. mRNA-encoding left and right TALENs designed to hit exon 3 of the apoBa gene (Supplemental Figure 1B) were injected into 1-cell-stage Tg(fli1:EGFP) embryos. We identified 3 mutations, out of which we chose to focus on an 8 bp deletion in the TALENs' target site, introducing a premature stop-codon after 70 amino acids (Supplemental Figure 1B). Both apoBa and apoBb.1 mutants displayed strong decreases of the respective mRNA levels (Figure 1E). In addition, Western blot analysis demonstrated complete absence of ApoB in apoBb.1 but not in apoBa mutants (Supplemental Figure $1 C)$, confirming previous data showing that ApoBb.1 is the predominant isoform ( $95 \%$ abundance; ref. 14$)$.

Characterization of the mutant phenotypes. apoBa homozygous mutants were viable, fertile, and morphologically indistinguishable from their WT siblings (Figure 1, F and H, and Supplemental Figure 2A). Moreover, lipid distribution was normal in these mutants, as shown by Oil Red O (ORO) staining (ref. 15 and Figure $1, \mathrm{G}$ and I). Similarly, injection of apoBa antisense morpholino oligonucleotides (MOs) did not elicit any noticeable phenotypes (Supplemental Figure 2, D and E). In contrast, $a p o B b .1$ homozygous mutants presented with several defects. First, they were easily identified due to their dark yolk (Figure 1J and Supplemental Figure 2B), indicative of impaired lipid absorption and aberrant accumulation of lipid droplets in the YSL $(15,21)$. This was also verified by ORO staining, which showed a strong reduction in circulating lipid levels (Figure $1 \mathrm{~K}$ ). Injection of apoBb.1 MOs into WT embryos fully phenocopied the mutant phenotype (Supplemental Figure 2F). However, no effects were observed following injection of apoBb.1 MOs into apoBb.1 mutants (Supplemental Figure 2, G and H), confirming the MO specificity. The central role of the apoBb.1 isoform was further corroborated by the decreased viability of the apoBb.1 mutants (Supplemental Figure $2 \mathrm{~L}$ ). In addition, we noticed that the few $a p o B b \cdot 1^{-1-}$ animals that survived by approximately $60 \mathrm{dpf}$ were significantly thinner than their WT counterparts (Supplemental Figure 2M). 
A
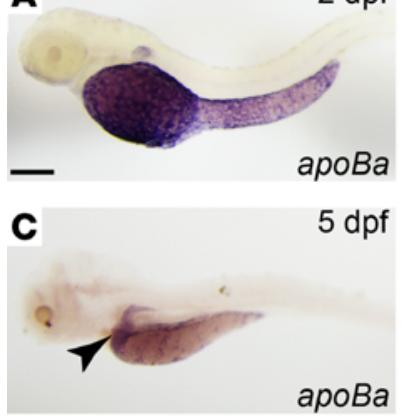

$2 \mathrm{dpf}$

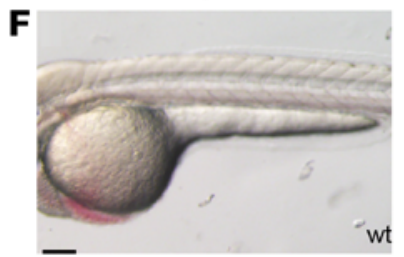

H

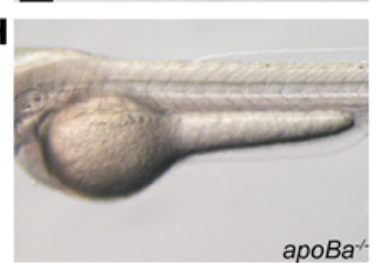

J

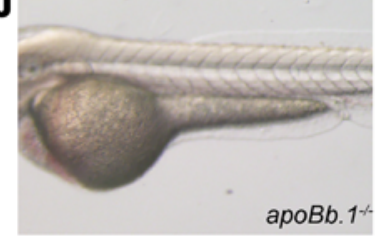

$\mathbf{L}$

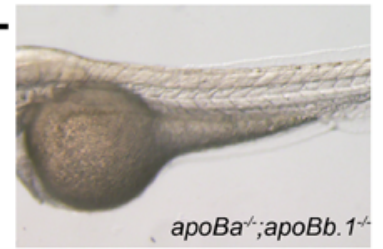

B

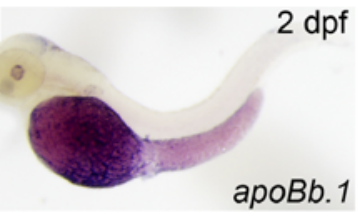

D

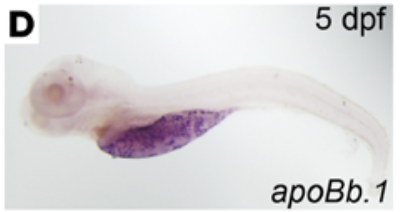

$3 \mathrm{dpf}$

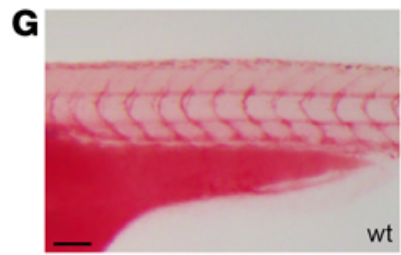

I

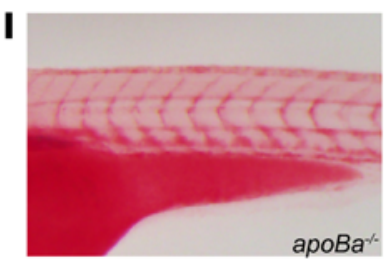

K

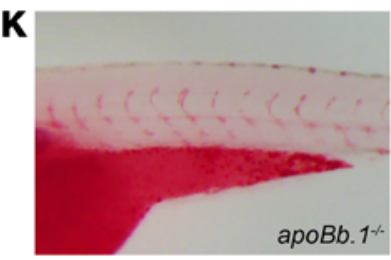

M

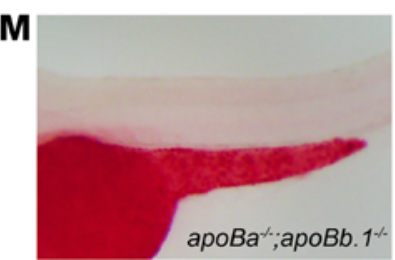

E

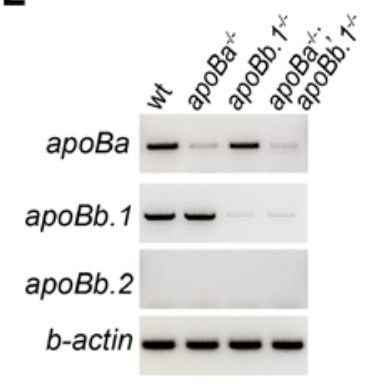

$\mathbf{N}$

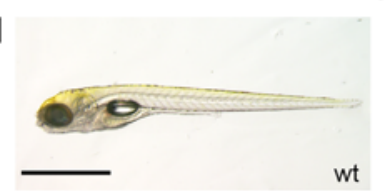

$6 \mathrm{dpf}$

O

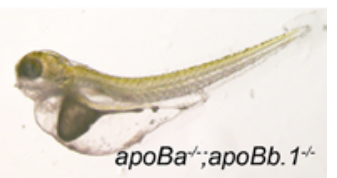

$\mathbf{P}$

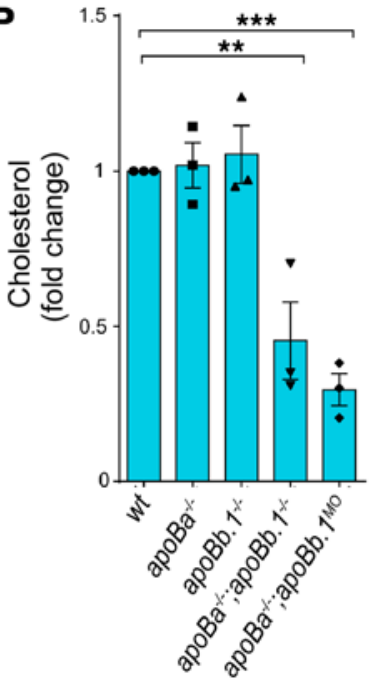

$\mathbf{Q}$

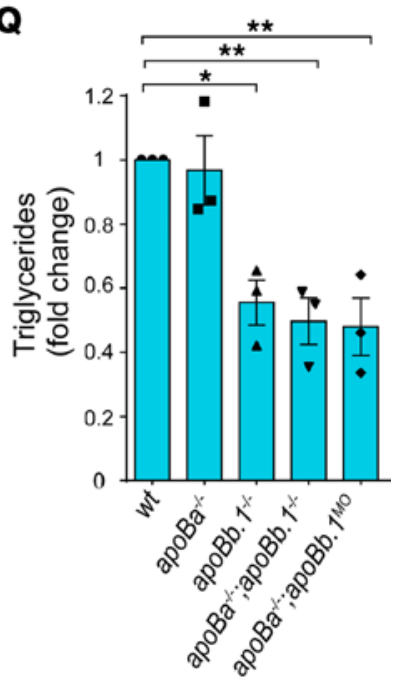

Figure 1. apoB mutants feature severe hypolipidemia. (A-D) Whole mount in situ hybridization (WISH) on 2 dpf zebrafish embryos showing strong expression of $a p o B a(\mathbf{A})$ and $a p o B b .1$ (B) in the YSL $\left(n_{\text {apoBa }}=7 ; n_{a p o B b .1}=6\right)$. At $5 \mathrm{dpf}$ apoBa expression is enriched in the liver (C) and apoBb.1 in the YSL and the intestine (D) ( $n_{\text {apoвa }}=3 ; n_{\text {apoBb. } 1}=3$ ). (E) Semiquantitative PCR for the $a p o B$ genes in the different mutants. (F-M) Transmitted light images of 2 dpf embryos showing dark yolk in $a p o B b .1^{-/-}(\mathbf{J})$ and $a p o B a^{-/-} a p o B b .1^{-/-}(\mathbf{L})$ but not in WT (F) or apoBa ${ }^{-/}(\mathbf{H})$ mutant embryos. Three dpf embryos stained with Oil Red O (ORO) show decreased lipid levels in apoBb.1 mutant (K), as compared with WT (G) and apoBa ${ }^{-1-}$ (I), embryos. (M) apoBa apoBb.1 double mutants display complete absence of lipids in circulation. ( $\mathbf{N}$ and $\mathbf{O}$ ) Transmitted light images at $6 \mathrm{dpf}$ demonstrate severe malformations, unabsorbed yolk, and pronounced edema in apoBa apoBb.1 double mutants as compared with WT siblings. (P and $\mathbf{Q})$ Cholesterol (P) and TC (Q) levels in the different apoB mutants compared with WT controls at $3 \mathrm{dpf}$. All measurements were carried out in deyolked embryos. $n=3, n$. $=20$. The data are shown as the mean \pm SEM, calculated using ANOVA followed by Tukey's multiple-comparison test. Scale bar: (A-D; F-M) $100 \mu$ m, ( $\mathbf{N}$ and $\mathbf{0}) 1 \mathrm{~mm}$. ${ }^{*} P<0.05,{ }^{* *} P<0.01$, ${ }^{* *} P<0.001 . P<0.05$ (considered significant versus control group).

Although ApoBb.1 represents the predominant ApoB isoform, we suspected that the presence of ApoBa in apoBb. 1 mutants could compensate for the lack of its functions. Therefore, we generated apoBa apoBb.1 double homozygous mutants (Figure 1L and Supplemental Figure 2C). These animals displayed a darker yolk and complete absence of lipids in circulation (Figure 1M). Moreover, the lack of both ApoB isoforms led to lethality at 6 to $8 \mathrm{dpf}$, with the larvae exhibiting profound yolk and pericardial edema, as well as a curved trunk (Figure 1, N and O). Finally, injection of apoBb.1 MOs into apoBa homozygous mutants or vice versa (apoBa MOs into apoBb. $1^{-/-}$embryos) recapitulated the phenotypes of apoB double mutants (Supplemental Figure 1C and Supplemental Figure 2, I-K). 
We then carried out a lipid profile analysis of the different mutants. In order to accurately measure yolk-to-body ApoB-dependent transport, we utilized deyolked embryos. As seen in Figure 1P, we found no differences in cholesterol levels between $a p o B a^{-1}$ and $a p o B b .1^{-/-}$individual mutants. In contrast, these were strongly reduced in both $a p o B a$ apoBb.1 double mutants and $a p o B a^{-/} a p o B b .1^{M O}$, as compared with WT siblings (Figure 1P). Interestingly, triglyceride (TG) measurements revealed a slightly different picture (Figure 1Q). While in apoBa ${ }^{-/}$mutants TG levels were similar to those of WT siblings, levels were strongly decreased in $a p o B b .1$ mutants (Figure 1Q). In addition, TG levels were significantly reduced in both apoBa apoBb.1 double mutants and in $a p o B a^{-/-} a p o B b .1^{M O}$ (Figure 1Q).

Due to the difficulty in obtaining large numbers of double homozygous mutants from apoBa ${ }^{-/-} \times$ $a p o B b .1^{+/-}$crosses, and having verified that the $a p o B b .1 \mathrm{MO}$ fully recapitulates the mutant phenotype, we decided to use apoBa ${ }^{-/}$apoBb. $1^{M O}$ embryos in most experiments of this study.

Defective organ development in $a p o B$ mutants. Next, we analyzed the development of the liver and the intestine, the main organs responsible for ApoB synthesis, assembly, and secretion in vertebrates (3). We took advantage of the fact that, in contrast to ApoB-null mice, apoBa ${ }^{-/-} a p o B b .1^{-/-}$double mutants survive beyond the initial stages of formation of the digestive system to investigate the role of ApoB during normal formation of these organs. To examine liver morphology, we used $T g(-2.8 f a b p 10 a: E G F P)^{a s} 3$, a well-established transgenic reporter expressing EGFP under the regulation of the fatty acid-binding protein $10 \mathrm{~A}$ (fabp10a) promoter (22). Injection of apoBb.1 MOs into $\mathrm{Tg}(f a b 10 a: E G F P)$ apoBa ${ }^{-1-}$ embryos resulted in significantly reduced liver size at $4 \mathrm{dpf}$ (Figure 2A and Supplemental Figure 3A), which was not due to apoptotic cell death, as confirmed by TUNEL staining (Figure 2B). Similar results were obtained following in situ hybridization for fabp 10a (Figure 2C), suggesting a defect in hepatocyte specification, differentiation, and/ or proliferation. To distinguish between these possibilities, we analyzed the expression of prospero homeobox 1a (prox1a), a transcription factor essential for hepatocyte differentiation and liver development (23, 24). As seen in Figure 2D, prox $1 a^{+}$hepatic progenitors were normally detected to the left of the midline at 2 $\mathrm{dpf}$, in phenotypically normal $a p o B a^{-/-}$embryos. By contrast, loss of both apoBa and apoBb. 1 caused embryos to develop bilateral livers (Figure 2D, arrowheads), suggesting an early role for ApoB in controlling liver laterality. This defective positioning of hepatoblasts was still detected at $4 \mathrm{dpf}$, as depicted by the expression of transferrin ( $t f a$ ), an additional liver-specific marker (ref. 25 and Figure 2E). Interestingly, these defects were specific for the liver because we did not detect laterality problems in gut looping (Figure 2F, arrowheads, and Supplemental Figure 3B) or in early left-right asymmetry markers, such as leftyl (not shown). These results indicate that hepatoblast specification is initiated normally in apoB-mutant embryos but that liver formation begins to be affected after 2 days of development.

Liver organogenesis in zebrafish involves 2 main phases: first, specification and migration of $h h_{e} x^{+}$ and prox $1^{+}$hepatoblasts to form the liver bud on the left side of the embryo, and second, hepatoblast differentiation into hepatocytes and biliary epithelial cells (BECs), followed by massive cell proliferation (26). To check whether the development of the intrahepatic biliary network is also affected in apoB mutants, we used the $T g$ (EPV.Tp1-Mmu.Hbb:EGFP) ja12 reporter (12xNRE:EGFP), which consists of 12 repeats of Notch-responsive elements driving EGFP expression (27), because Notch signaling in hepatic progenitor cells was shown to be both required and sufficient for biliary specification (28). At $5 \mathrm{dpf}$, the $\operatorname{Tg}(12 \times \mathrm{N}$ $R E: E G F P$ ) positive intrahepatic biliary network was highly branched in WT, apoBa ${ }^{-/}$, and apoBb.1 $1^{-/-}$larvae (Figure $2 \mathrm{G}$ ), as opposed to $a p o B a^{-/-} a p o B b .1^{M O}$ that displayed largely reduced Notch activity. Taken together, the observed phenotypes suggest that, while $\mathrm{ApoB}$ is not required for initial hepatocyte specification, it appears to play an important role in hepatoblast positioning (i.e., budding and/or migration), proliferation, and segregation between hepatocytes and BECs.

Hepatic steatosis, the accumulation of lipid within hepatocytes, is a critical step in the pathogenesis of several human diseases, including alcoholic liver disease and NAFLD. While NAFLD is mostly associated with hyperlipidemia (29), defective synthesis and secretion of ApoB lipoproteins can also lead to hepatic steatosis, as observed in patients with FHBL1 $(9,30)$. To investigate whether the different apoB mutants develop steatosis, we utilized Nile red staining, which labels polar lipids in red and neutral lipids in green $(31,32)$. As expected, liver lipid stores were nearly undetected in WT and apoBa $a^{-/}$mutants (Figure $\left.2 \mathrm{H}\right)$, as opposed to apoBb.1 $1^{M O}$, which featured excessive accumulation of lipid droplets in hepatocytes (Figure $2 \mathrm{H}$ ). Interestingly, lipid droplet accumulation was less severe in the livers of $a p o B a^{-1} a p o B b .1^{M O}$ embryos (Figure $2 \mathrm{H}$ ), most probably due to the impaired YSL lipoprotein production (Figure $2 \mathrm{H}$, arrowheads). These results were confirmed by H\&E staining of histological sections (Supplemental Figure 3C, arrowheads). 
A
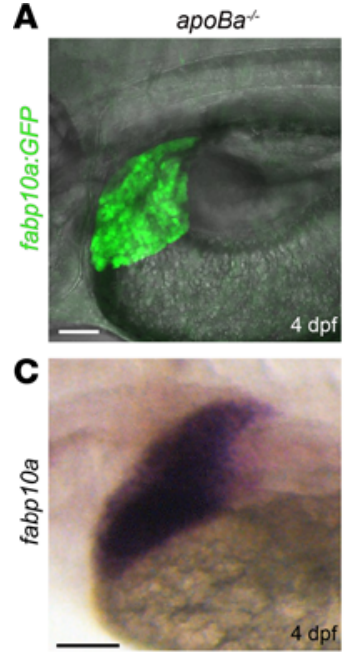

E

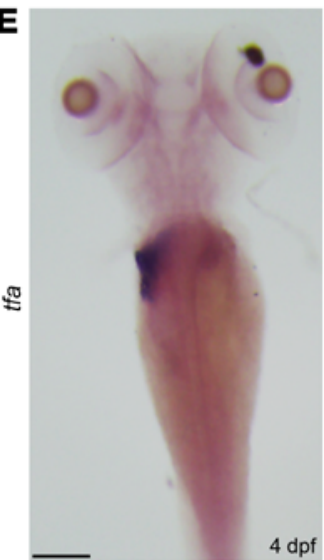

wt
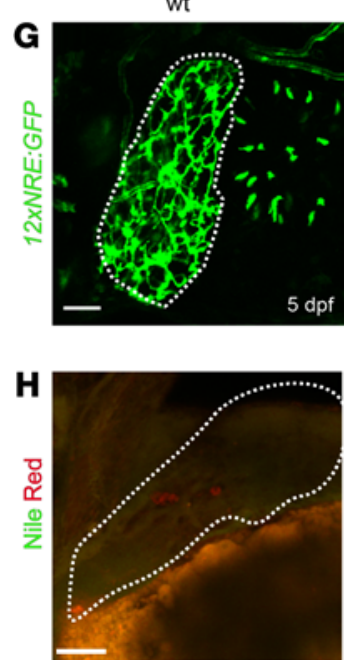

apoBa $; a p o B b .1^{\text {мо }}$
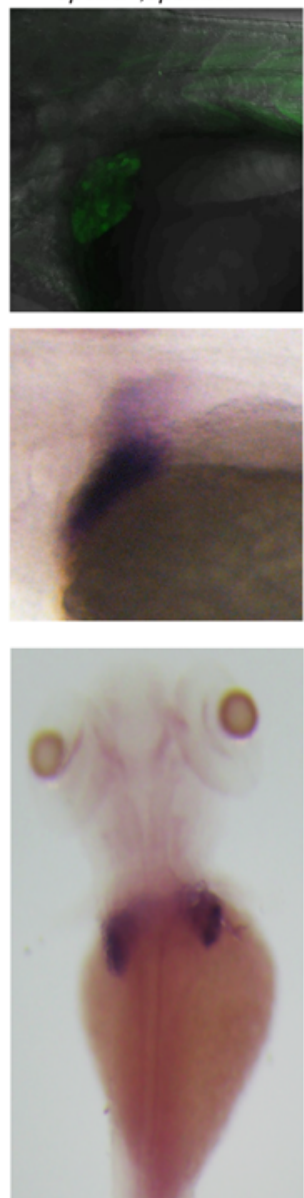

apoBa-
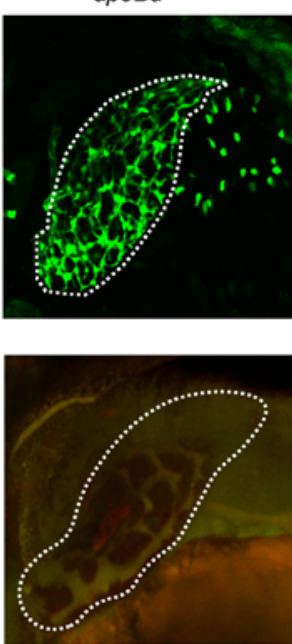

B
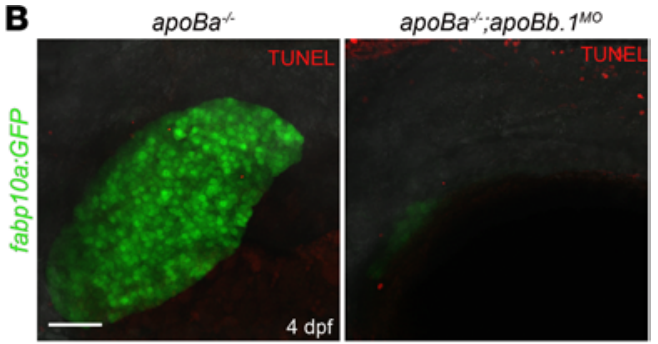

D
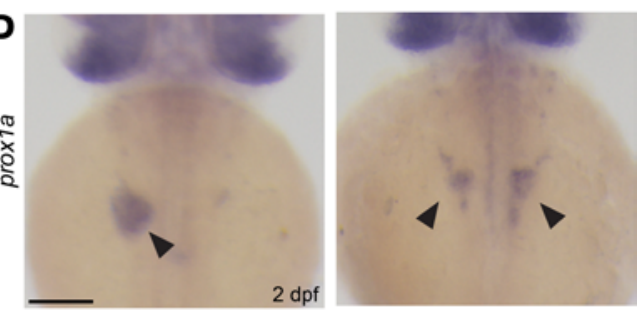

$\mathbf{F}$

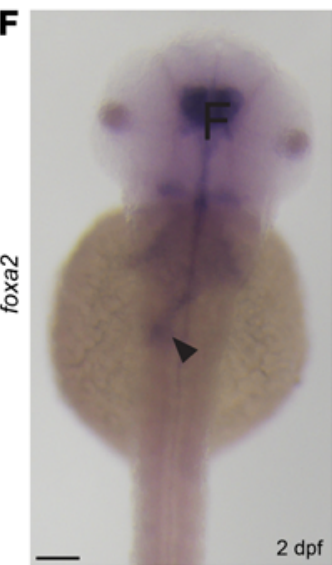

apoBb. $1^{\text {th }}$
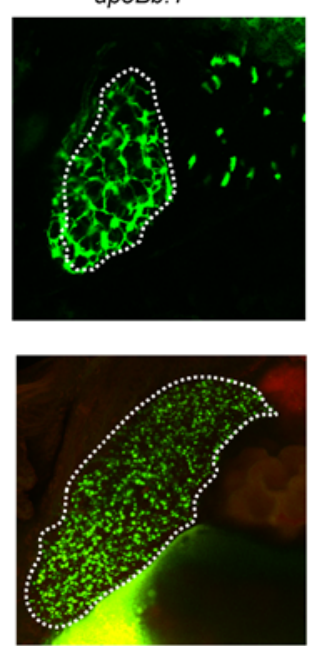

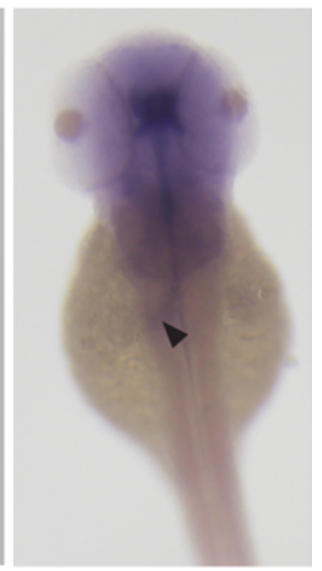

apoBa ;apoBb. $1^{\text {mo }}$
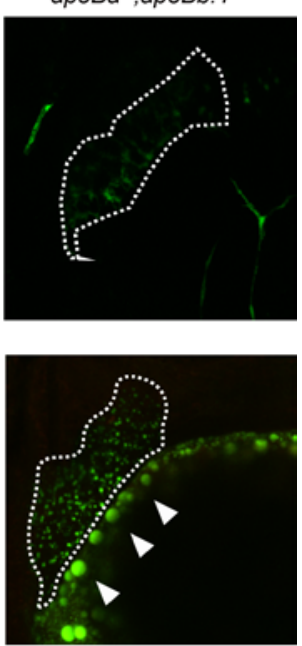

Figure 2. apoB mutants exhibit defective liver development and steatosis. (A) Confocal images at $4 \mathrm{dpf}$ of $a p o B a^{-1-}$ and $a p o B a^{-1-}$ apoBb. $7^{\mathrm{MO}}$ embryos in

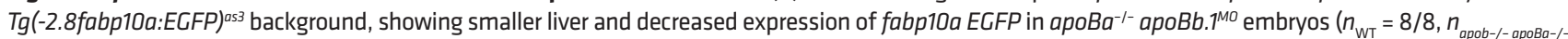

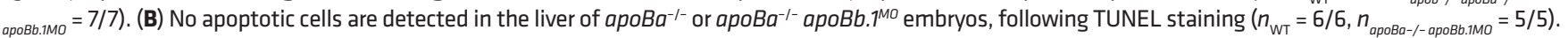
(C) WISH showing expression of fabp10a at $4 \mathrm{dpf}$, depicting decreased liver size in $a p o B a^{-/-}$apoBb. $7^{\mathrm{MO}}$ embryos $\left(n_{\mathrm{WT}}=7, n_{\text {apoBa- } / \text { apoBb.1MO }}=13\right)$. (D and E) WISH with

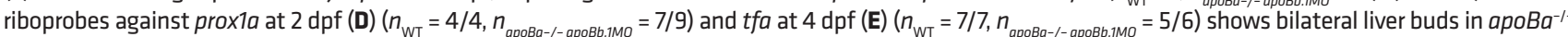
apoBb. $1^{\mathrm{MO}}$ embryos as opposed to apoBa-l- mutants, which display a single liver bud located to the left of the midline. (F) Expression of forkhead box a2 (foxa2) at $2 \mathrm{dpf}$ remains unchanged in $a p o B a^{-1-} a p o B b .7^{M 0}$ embryos as compared with apoBa ${ }^{-1-}$ siblings. (C) Confocal images of $5 \mathrm{dpf} \operatorname{Tg}(12 \times N R E$ :Egfp) embryos with

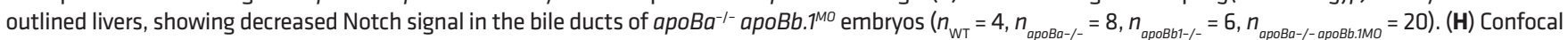
images of Nile red staining at $4 \mathrm{dpf}$ depicting neutral lipid accumulation in $a p o B b .7^{\mathrm{MO}}$ and $a p o B a^{-1-} a p o B b .7^{100}$ embryos. Red staining labels polar lipid; green fluorescence highlights neutral lipids $\left(n_{\mathrm{WT}}=5, n_{\text {apoBa-/- }}=8\right.$, napoBb.1-/-$\left.=5, n_{\text {apoBa-/- apoBb.1M0 }}=9\right)$. Scale bar: (A, B, G, and H) $50 \mu \mathrm{m},(\mathbf{C}-\mathbf{F}) 100 \mu \mathrm{m}$. 

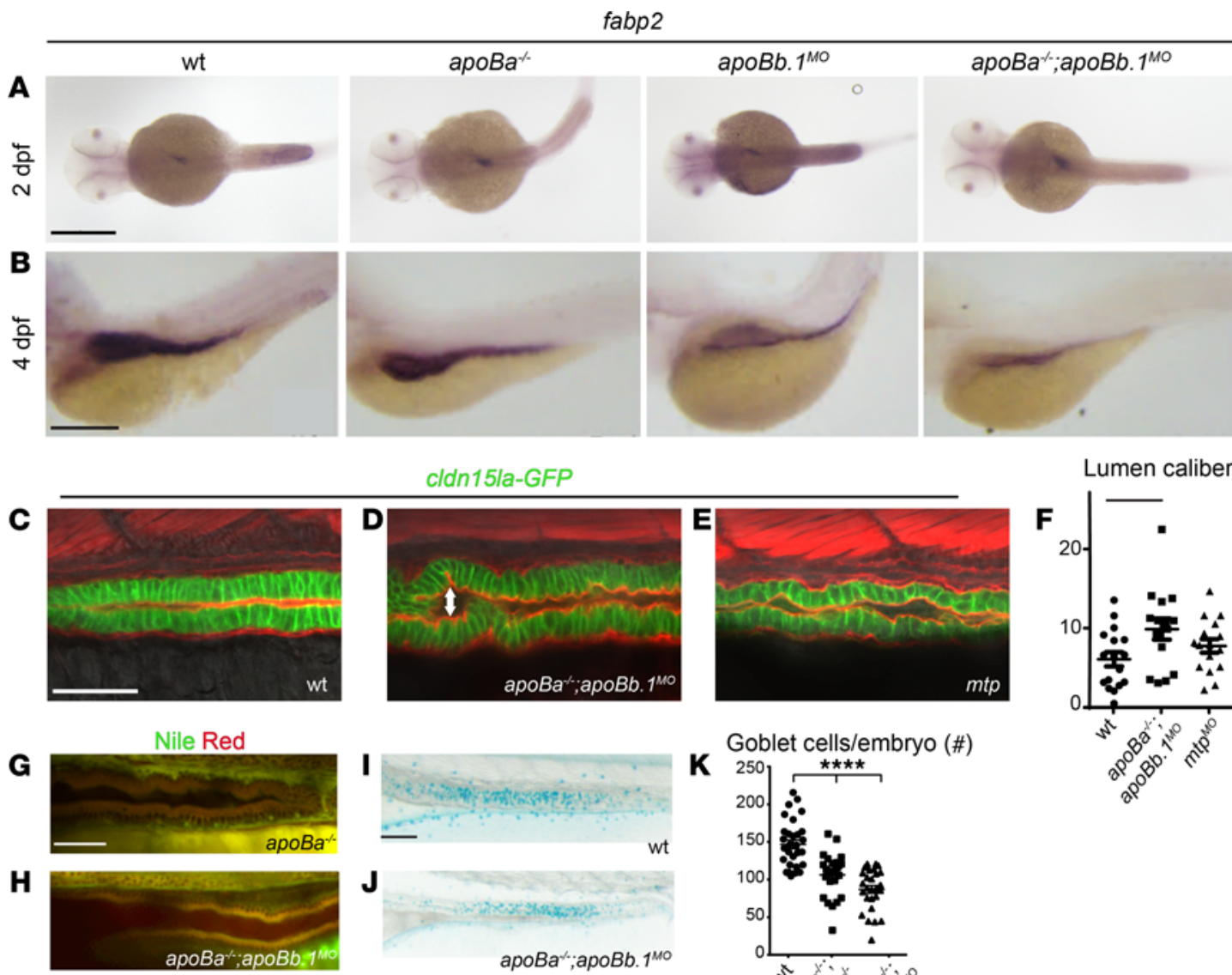

Lumen caliber

Goblet cells/embryo (\#)
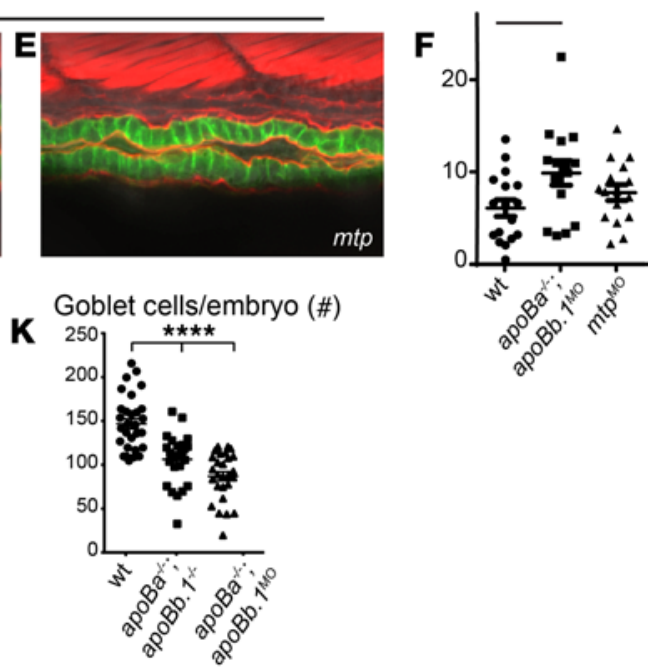

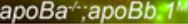

apoBa $; a p o B b .1^{\text {mo }}$

12XNRE:GFP

$\mathbf{L}$

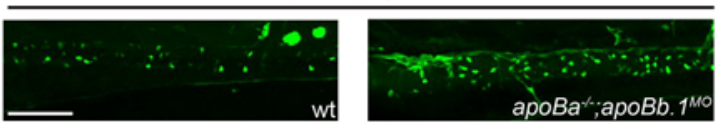

$\mathbf{M}$

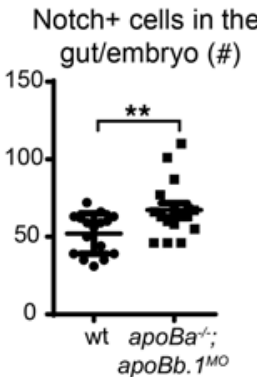

Figure 3. Gut development is impaired in apoB mutants. (A and B) WISH showing the spatial expression of fabp2 at 2 (A) and 4 (B) dpf. (C-E) Confocal images at $4 \mathrm{dpf}$ of WT (C), apoBa $a^{-/-} a p o B b .1^{\mathrm{MO}}(\mathbf{D})$, and $m t p^{\mathrm{MO}}(\mathbf{E})$ embryos in the TgBAC(cldn15la-GFP)pd1034 reporter background, stained with phalloidin (red). Enlarged lumen caliber (arrow) is detected in $a p o B a^{-/-} a p o B b .7^{M 0}$ and $m t p^{M 0}$ embryos, as quantified $(\mathbf{F})\left(n=2, n_{\text {WT }}=17, n_{\text {apoBa }-/- \text { apoBb.1MO }}=15, n_{m t p M O}=16\right)$. The data are shown as the mean \pm SEM, calculated using ANOVA followed by Tukey's multiple-comparison test. ${ }^{*} P<0.05$. (G and $\left.\mathbf{H}\right)$ Confocal images of the guts of $4 \mathrm{dpf} a p o \mathrm{Ba}^{-1-}$ and $a p o \mathrm{Ba}^{-1-}$ apoBb..$^{\mathrm{Mo}}$ embryos stained with Nile red, show no lipid accumulation. (I and J) Bright-field images of $5 \mathrm{dpf}$ embryos stained with Alcian blue showing reduced number of goblet cells in apoBa ${ }^{-1-}$ apoBb.1 $1^{-1-}$ double mutants $(\mathrm{J})$ as compared with WT embryos $(\mathbf{I})$, as quantified $(\mathbf{K})(n=3$,

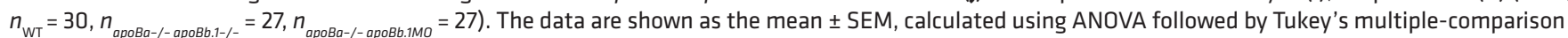
test. ${ }^{* * *} P<0.0001$. (L) Confocal images at $5 \mathrm{dpf}$ of WT and apoBa ${ }^{-/-}$apoBb.1 $7^{\mathrm{MO}}$ embryos in the $\mathrm{Tg}(12 \times N R E$ :Egfp) reporter background. (M) Quantification of number of cells displaying active Notch signaling in the guts of WT and apoBa ${ }^{-1-}$ apoBb. $7^{\mathrm{Mo}}$ embryos. The data are shown as the mean \pm SEM, calculated using 2-tailed Student's $t$ test. Scale bar: (A-E, G, and $\mathbf{H}) 50 \mu \mathrm{m},(\mathbf{I}, \mathbf{J}$, and $\mathbf{L}) 100 \mu \mathrm{m}$. ${ }^{*} P<0.01 . P<0.05$ (considered significant versus control group).

Because of the close relationship between liver and intestine formation, we tested whether ApoB depletion also affects gut development. The intestine is responsible for the absorption of lipids and their secretion in the form of chylomicrons into lymphatic vessels. Analysis of foxa2 (ref. 33; Figure 2F, arrowheads; and Supplemental Figure 3B) and fatty acid binding protein 2 ( fabp2) (ref. 25 and Figure 3A) expression revealed normal development of the intestinal tube in individual mutants at $2 \mathrm{dpf}$. However, by $4 \mathrm{dpf}$ we noticed that the gut appeared somewhat reduced in size in apoBa ${ }^{-/-} a p o B b .1^{M O}$ embryos (Figure $3 \mathrm{~B}$ ). Yet, the 
effects of ApoB depletion on gut formation appeared to be less severe than the effects observed in the liver. We also inspected the guts of the different mutants by mating the fish with the TgBAC(cldn15la-GFP) ${ }^{\text {pd } 1034}$ (34) reporter, which labels tight junctions in the intestine. We detected slightly enlarged lumens in the guts of apoBa $a^{-/}$apoBb. $1^{M O}$ embryos (Figure 3, C, D, and F) as compared with WT siblings. Similar effects were observed following injection of $m t p$ MOs (Figure 3, C, E, F). These defects were corroborated by direct inspection of H\&E staining of histological sections (Supplemental Figure 3D). Although at this stage of development the intestine is known to be folded and to contain polarized epithelial cells with a microvillus brush border (ref. 35 and Supplemental Figure 3D), in apoBa ${ }^{-/} a p o B b .1^{-/-}$embryos, the intestinal epithelium appeared thin and poorly folded, and the cells had only few scattered microvilli as compared with their WT counterparts (Supplemental Figure 3, D and E, blue arrowheads). Also, intestine-specific deletion of Mtp or $A p o B$ in mice renders small and large intestines with enlarged calibers $(36,37)$, mostly due to fat accumulation in the villus enterocytes, fully recapitulating the phenotypes reported for abetalipoproteinemia (38). In contrast, we did not detect lipid accumulation in enterocytes of $a p o B a^{-1} a p o B b .1^{-/-}$mutants (Figure 3, G and $\mathrm{H}$ ), suggesting that the enlarged lumen phenotype and thinner epithelia are most likely derived from impaired nutrient absorption, as previously described (39).

Finally, Alcian blue staining at $5 \mathrm{dpf}$ highlighted a significantly reduced number of goblet cells in apoBa-1apoBb. $1^{-/-}$mutant guts (Figure 3, I-K). Previous reports have shown that inhibition of Notch signaling directs the specification of intestinal cells toward a secretory fate (40), leading to increased numbers of goblet cells. We therefore evaluated the levels of Notch activation in the guts of WT and mutant embryos using the 12NRE:GFP reporter. As seen in Figure 3, L and M, we detected increased numbers of $\mathrm{Notch}^{+}$cells in the guts of apoBa ${ }^{-/} a p o B b .1^{M O}$ embryos as compared with WT siblings, pointing to alteration in Notch signaling as the mechanism underlying goblet cell differentiation. Altogether, our results indicate that ApoB deficiency results in impaired liver development accompanied by hepatic steatosis and disrupted intestinal architecture.

$A p o B$ depletion affects vascular development. Previous studies, including our own, have demonstrated that ApoB-containing lipoproteins have an inhibitory effect on vascular growth $(2,15,16,41)$. We therefore decided to analyze the involvement of the ApoB isoforms in the development of the vascular system by assessing the early vasculature of the different mutants. We first focused on the subintestinal vessels (SIVs), a plexus located on top of the YSL (Figure 4A) that plays a critical role in yolk absorption and vascularization of the gastrointestinal tract $(15,27,42)$. In line with the lipid and morphological defects, the vascular phenotypes were more pronounced in the double homozygous mutants, as compared with WT siblings or single mutants. As seen in Figure 4, B-D and H-I, the SIVs formed normally in apoBa ${ }^{-1-}$ and displayed only minor defects in apoBb.1 mutants. By contrast, apoBa apoBb.1 double mutants (Figure 4, E, H, and I) and $a p o B a^{-/}$apoBb. $1^{M O}$ (Figure 4, $\mathrm{F}, \mathrm{H}$, and I) larvae exhibited ectopic angiogenic sprouts arising in the SIVs, which did not retract during the remodeling phase as they did in WT siblings (27). These sprouts, reminiscent of those observed in stl mutants (Figure 4G and ref. 15), were clearly detected following injection of apoBb.1 MOs into WT embryos (Figure 4, E, F, H, and I). However, no effects were observed upon injection of apoBb.1 MOs into apoBb.1 mutants (Supplemental Figure 4, B-E), confirming the MO specificity. Similar hyperangiogenic behaviors were detected in the posterior cerebral vein $(\mathrm{PCeV})$ at $4 \mathrm{dpf}$ (Supplemental Figure 4, A and F-H), and in the dorsal trunk (Supplemental Figure 4, A and I-K) of $a p o B a^{-/-} a p o B b .1^{M O}$ larvae, suggesting that impaired angiogenesis is a general consequence of $\mathrm{ApoB}$ depletion that becomes more pronounced as development proceeds.

We have previously shown that $\mathrm{ApoB}$ lipoproteins induce ectopic angiogenesis by downregulating the expression of the VEGF decoy receptor Vegfr1/Flt1 (15). Accordingly, depletion of both membranous and soluble forms of Flt1 fully phenocopied this phenotype (27). Given that loss of Flt1 has been shown to result in enhanced Notch signaling (43), and that $\mathrm{Flt}^{-/-}$mutant sprouts are less likely to retract (44), we decided to investigate whether this mechanism is activated in $a p o B$ mutants. Live imaging of the $12 x N R E$ :EGFP reporter revealed clear activation of Notch signaling in the ectopic sprouts that failed to retract in $a p o B a^{-/} a p o B b .1^{M O}$ embryos (Figure 4, J and K). In contrast, no ECs displaying active Notch signaling were detected in the SIV plexus of WT embryos at comparable developmental stages (i.e., $5 \mathrm{dpf}$ ), when the vessels have acquired their final, quiescent stage. Taken together, these results reinforce and expand our previous findings by showing that not only Flt1 but also Notch signaling act downstream of ApoB to modulate angiogenesis.

In order to verify that the observed vascular phenotypes indeed derive from the absence of ApoB lipoproteins, we attempted to rescue the defects by intravascularly injecting DiI-labeled LDL into apoBa ${ }^{-1-} a p o B b$. $1^{\text {MO }}$ embryos (Supplemental Figure $4 \mathrm{~L}$ ) as previously described $(15,17)$. As seen in Figure $4, \mathrm{~L}-\mathrm{N}$, a single injection of DiI-LDL was sufficient to revert the excessive angiogenesis phenotype, as opposed to oleic acid 
A
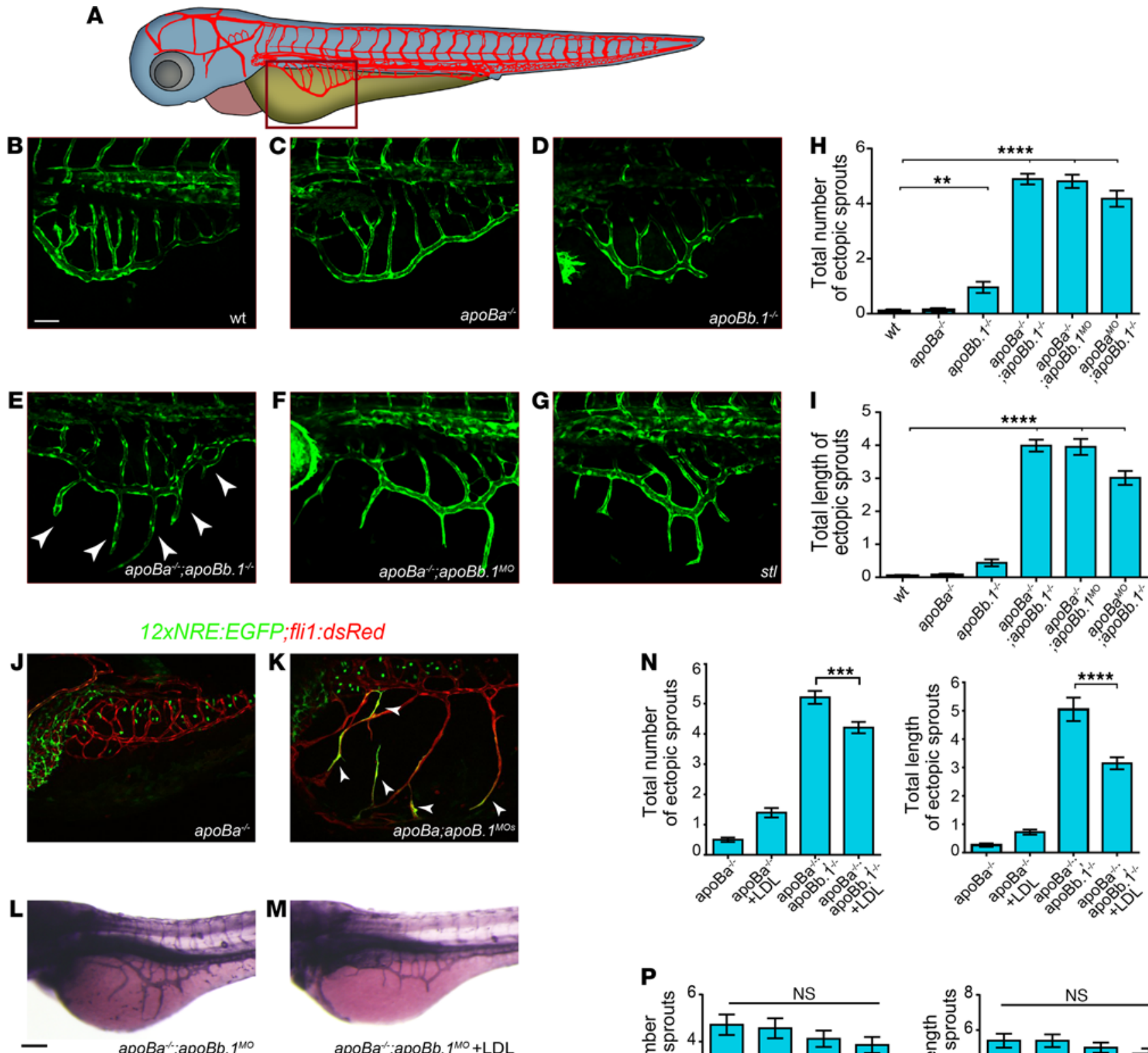

apoBar;apoBb. $1^{\mathrm{MO}}+\mathrm{LDL}$
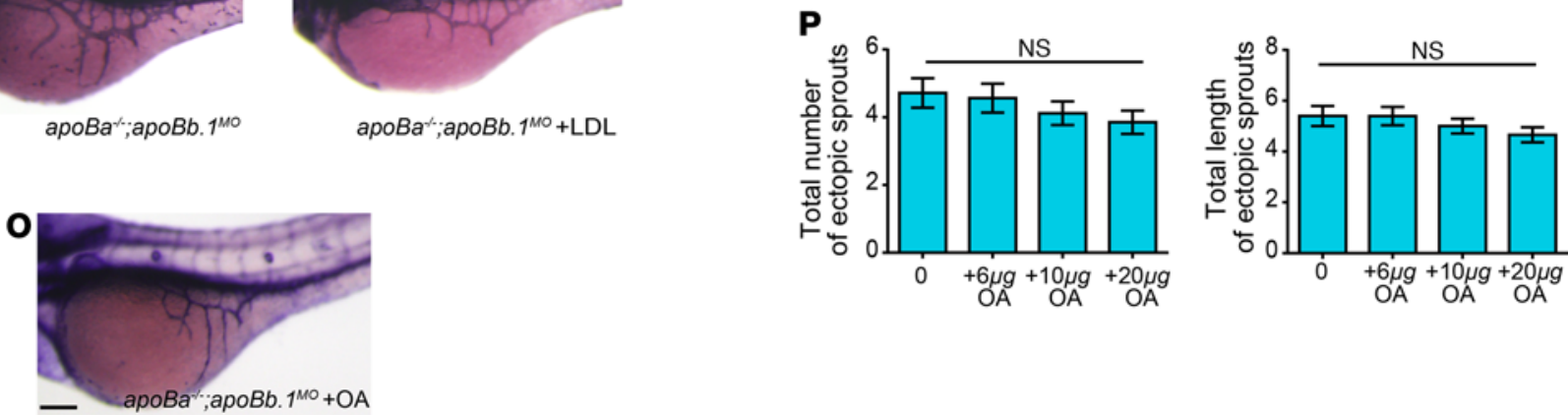

Figure 4. ароB mutants display hyperangiogenic phenotypes. (A) Schematic representation of the zebrafish embryonic vasculature, with red square marking the subintestinal vessels (SIVs). (B-C) Confocal images at $3 \mathrm{dpf}$ showing ectopic sprouts (arrowheads) arising in the SIVs of apoba ${ }^{-1-}$ apoBb..$^{-1-}$ double

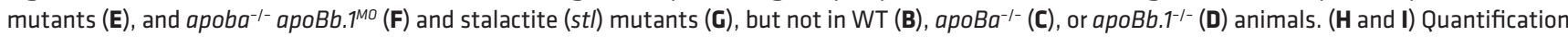

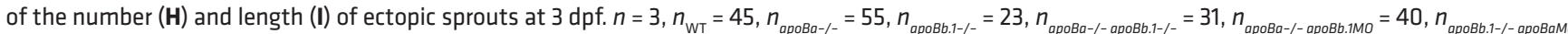
$=21$. The data are shown as the mean \pm SEM, calculated using ANOVA followed by Tukey's multiple-comparison test. ${ }^{* * *} P<0.0001$, ${ }^{* *} P<0.01$. (J and $\mathbf{K}$ ) Confocal images at $5 \mathrm{dpf}$ of $a p o B a^{-/-}$and $a p o b a^{-/-}$apoBb. $1^{\mathrm{MO}}$ embryos in the $\mathrm{Tg}\left(12 \times N R E\right.$ :Egfp) reporter background. White arrowheads in apoBa ${ }^{-/-} a p o B b .7^{\mathrm{MO}}$

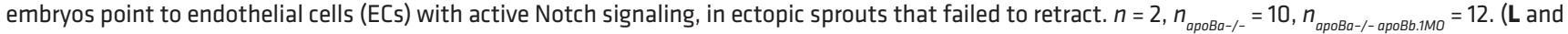
M) Alkaline phosphatase (AP) staining of the SIVs at $3 \mathrm{dpf}$, showing inhibition of ectopic sprouting following intravascular injection of Dil-LDL into apoBa-1 $a p o B b .7^{M 0}$. (N) Quantification of number and length of ectopic sprouts following intravascular injection of Dil-LDL $\left(n=3, n_{\text {apoBa- }}=57, n_{\text {apoBa- }- \text { - }+\mathrm{LDL}}=43, n_{\text {apoBa-//- }}\right.$ $\left.{ }_{\text {apoBb.1Mo }}=67, n_{\text {apoBa- } / \text { apoBb.1MO+LDL }}=57\right)$. The data are shown as the mean \pm SEM, calculated using ANOVA followed by Tukey's multiple-comparison test. ${ }^{* * * *} P$

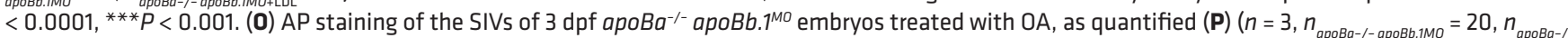

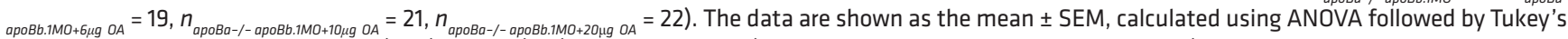
multiple-comparison test. Scale bar: (B-G) $50 \mu \mathrm{m},(\mathbf{J}-\mathbf{0}) 100 \mu \mathrm{m} . P<0.05$ (considered significant versus control group). 
(OA) supply (Figure 4, O and P), supporting a specific role for ApoB, and not the lipid moieties within lipoproteins, in developmental angiogenesis. These results are in line with our previous findings, which showed that the strong excessive angiogenesis phenotype stl mutants displayed in response to lipoprotein depletion (Figure 4G) was efficiently reversed following injection of a delipidated form of ApoB (15).

Because this pathway could potentially be exploited to control pathological neovascularization, we proceeded to define the domains within ApoB that act on ECs. To this end, we tested the ability of truncated forms of the human APOB protein to inhibit angiogenesis, using the well-established tube formation assay. To ensure that lipoproteins are presented to the cells in their physiological form, we cotransfected MTP along with human APOB34 or APOB25, which have been previously shown to undergo proper lipidation and secretion (45, 46), into HEK293 cells (Supplemental Figure 4M). We then transferred the media containing the different truncated forms to human umbilical vein endothelial cells (HUVECs) and examined their ability to generate tubes. LDL, which we previously showed inhibits tube formation in culture (16), was used as positive control. Interestingly, both APOB25 and APOB34 forms inhibited angiogenesis in this assay, suggesting that the antiangiogenic effect of ApoB is conveyed by the $25 \%$ (N-terminus) of the protein (Figure 5, A-E).

We then asked whether these fragments are sufficient to inhibit angiogenesis in vivo. To answer this question, we generated plasmids expressing human $A P O B 25$ and $A P O B 34$ fused to IRESmCherry, under the regulation of the apo14 promoter, which drives expression in the YSL and the liver (47). While the hyperangiogenic phenotype of apoBa ${ }^{-1-}$ apoBb.1 $1^{M O}$ embryos was not reverted following apo14:APOB25-IRESmCherry injection (Figure 5, F-H, J, and $\mathrm{K}$ ), apoBa ${ }^{-/-}$apoBb.1 $1^{M O}$ embryos injected with apo14:APOB34-IRESmCherry displayed a significant reduction of both the number and length of the ectopic sprouts at $4 \mathrm{dpf}$ (Figure 5, G, I, L, and M). These results suggest that the first $34 \%$ of the ApoB protein is required to exert its antiangiogenic effect in vivo. The discrepancy between the in vivo and the in vitro results may derive from differences in the mobility and clearance of the truncated forms of APOB in vivo. Specifically, it has been shown that APOB25 is not present in circulation in vivo, despite it being produced and secreted into the blood $(45,48)$, due to fast clearance.

As a whole these results demonstrate the applicability of our newly generated models of ApoB deficiency for assessing the physiological activity of potential therapeutic agents.

\section{Discussion}

In this work, we generated zebrafish mutants for $2 \mathrm{ApoB}$ isoforms: $a p o B a$ and $a p o B b .1$. Similar to $A p o B$ null mice, zebrafish carrying mutations in both $a p o B a$ and $a p o B b .1$ demonstrate early lethality. However, in contrast to the mammalian models, the external fertilization and development of zebrafish allowed us to investigate previously unappreciated roles of ApoB during embryogenesis.

Three $a p o B$ genes are present in the zebrafish genome, namely $a p o B a, a p o B b .1$, and $a p o B b .2$. During early development, $a p o B a$ and $a p o B b .1$ are highly expressed, whereas $a p o B b .2$ is absent (14). Interestingly, despite the strong expression of apoBa mRNA, its protein levels are almost 20-fold lower than those of ApoBb.1 (14). Accordingly, while the absence of $a p o B a$ did not elicit any noticeable phenotypes, $a p o B b .1$ depletion caused strong hypolipidemia. Finally, deletion of both isoforms resulted in severe phenotypes and lethality.

Our results uncover the roles for ApoB during development of the liver, one of its main producing organs. In particular, we find that $a p o B a^{-1} a p o B b .1^{M O}$ embryos display similar phenotypes to those caused by retinoic acid (RA) deficiency (49), including decreased liver volumes and liver bud bilaterality. Zebrafish embryos treated with the Raldh inhibitor DEAB, or injected with MOs against the 3 RA receptors (rarab, raraa, and rarga), were shown to display smaller livers because of impaired hepatocyte proliferation. In contrast, RA addition to the fish water resulted in massive proliferation of hepatocytes and enlarged livers (49). In addition, downregulation of the RA receptor rargb led to bilateral liver buds and intrahepatic biliary defects, similar to those displayed by double $a p o B$ mutants. While most RA circulation is mediated through retinol binding protein 4, it has been shown that ApoB-containing lipoproteins (e.g., LDL and VLDL) also participate in RA shuttling (50). Therefore, the possibility exists that complete depletion of ApoB leads to RA unavailability, which in turn results in liver bilaterality and reduced hepatocyte and BEC proliferation.

The absence of both apoB isoforms resulted in low levels of TGs and cholesterol in circulation, accompanied by massive lipid droplet accumulation in hepatocytes, and liver steatosis. These phenotypes, reminiscent of human NAFLD, recapitulate part of the clinical manifestations of FHBL1, making the apoBa ${ }^{-{ }_{-}}$apoBb.1 $1^{-{ }^{--}}$mutant zebrafish an advantageous model for the study of this disorder and for the identification of potential treatments.

In addition to liver abnormalities, ApoB and MTP deficiency resulted in disrupted intestinal architecture. In particular, the guts were poorly folded and displayed enlarged lumen calibers, resembling phenotypes displayed 
A
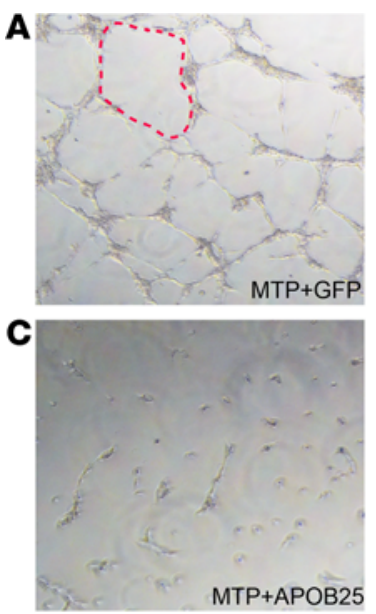

fli:EGFP;apoBa*
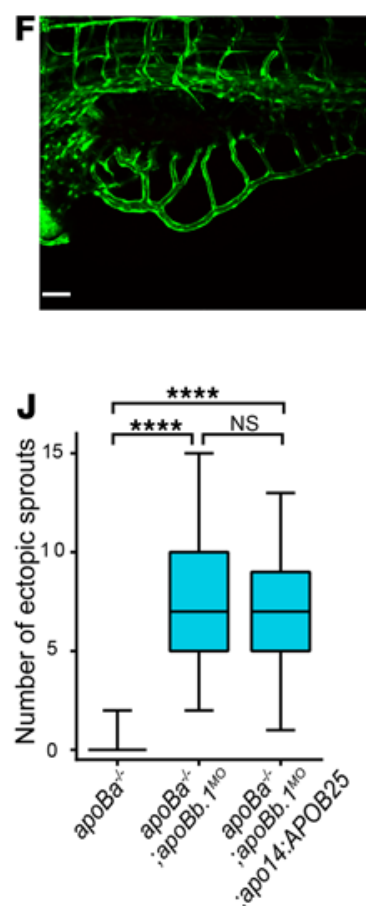

B
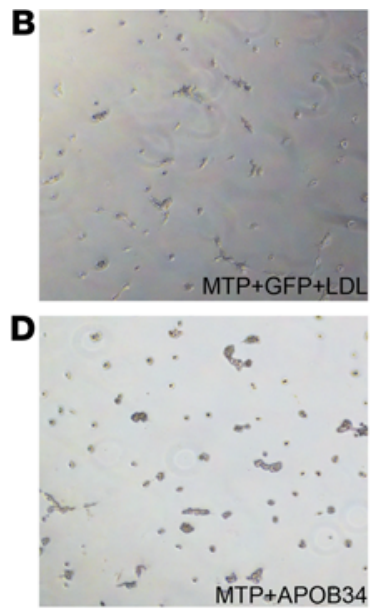

E

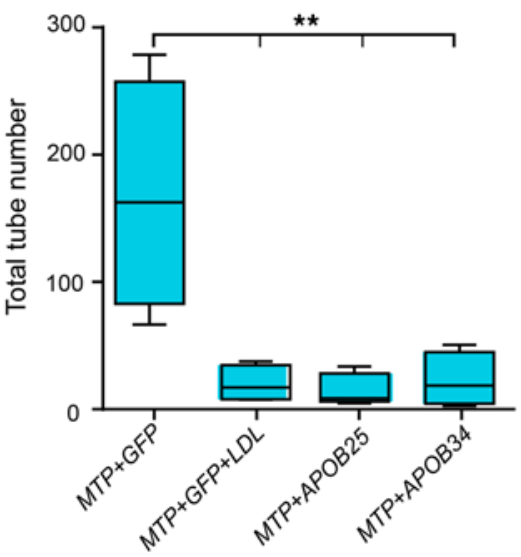

fli:EGFP;apoBa $\% ; a p o B b .1^{\text {Mo }}$
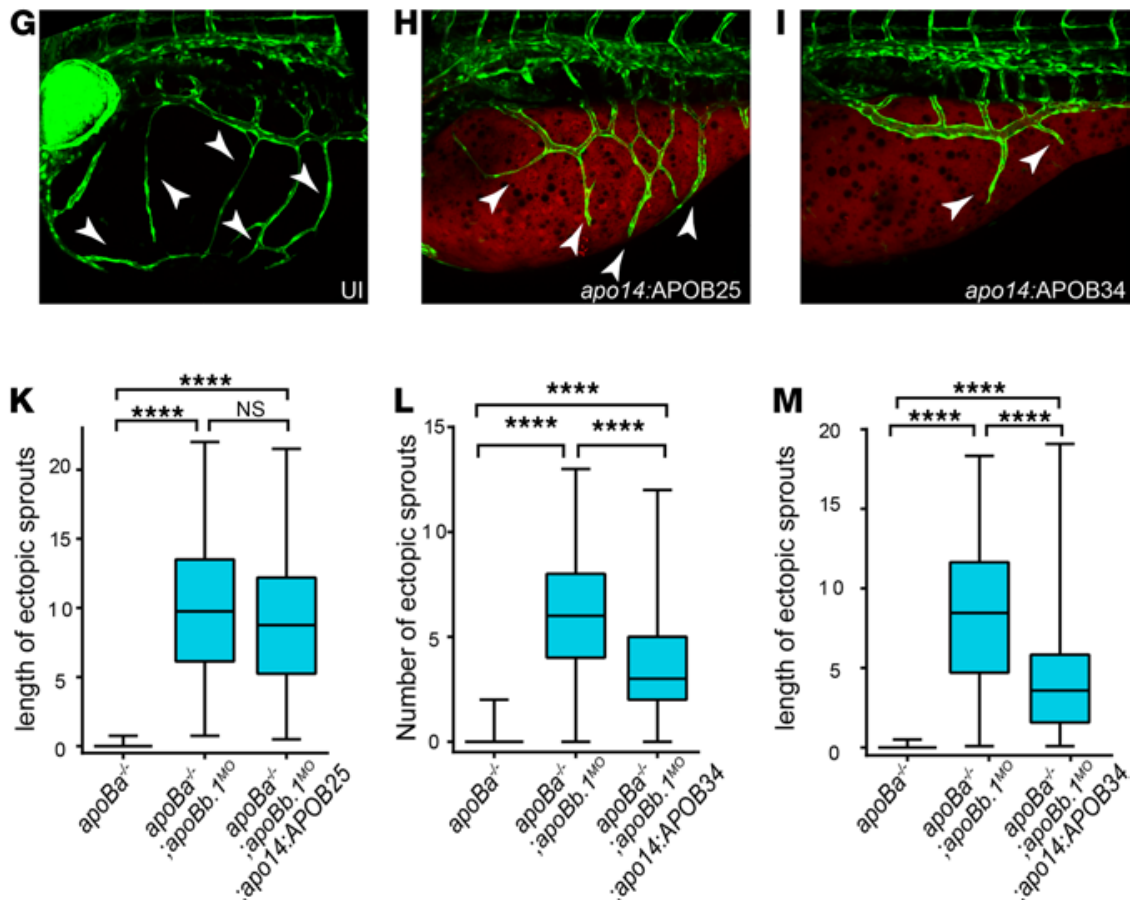

Figure 5. Truncated forms of human APOB inhibit angiogenesis in apoB mutants. (A-D) Tube formation assay on HUVECs untreated (A, C, and D) or treated with LDL (B) plus conditioned medium from HEK293 cells cotransfected with (A and B) MTP+GFP, (C) MTP+APOB25, and (D) MTP+APOB34. Red dashed line outlines vascular tube structure. (E) Quantification of total number of tubes following different treatments $\left(n=3, n_{M T P+C F P}=4, n_{M T P+C F P+L L L}=4, n_{M T P+A P O B 25}=5, n_{M T P+A P O B 34}=\right.$ 4). The data are shown as the mean \pm SEM, calculated using ANOVA followed by Tukey's multiple-comparison test. ${ }^{* *} P<0.01$. (F-I) Confocal images at 4 dpf showing reduction in the number and length of ectopic sprouts in Tg(flit:EGFP) apoBa ${ }^{-/-}$apoBb..$^{\mathrm{Mo}}$ embryos injected with apo14:APOB34 (I) as compared with uninjected $a p o B a^{-/-}(\mathbf{F})$, uninjected $a p o B a^{--} a p o B b .7^{\mathrm{MO}}(\mathbf{C})$, and $a p o B a^{-/-}$apoBb. $7^{\mathrm{MO}}$ injected with apo14:APOB25 (H). White arrowheads point to ectopic sprouts.

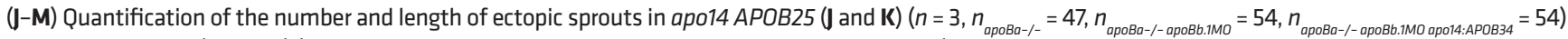

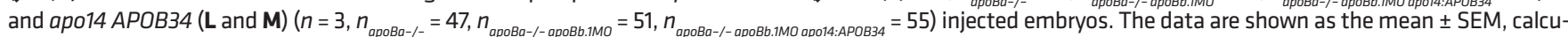
lated using ANOVA followed by Tukey's multiple-comparison test. Scale bar (F-I): $50 \mu \mathrm{m}$. ${ }^{* * *} P<0.0001 . P<0.05$ (considered significant versus control group).

by starved WT larvae $(39,51)$. Double $a p o B$ mutants feature an increased number of goblet cells. Interestingly, it has been shown that RA negatively regulates goblet cell differentiation (52), whereas inhibition of Notch signaling directs specification of intestinal cells toward a secretory fate (40) leading to increased numbers of goblet cells. Our results indeed demonstrate increased activation of Notch signaling in the intestines of apo$B a^{--} a p o B b .1^{-1-}$ mutants, supporting the idea that $\mathrm{ApoB}$ affects goblet cell differentiation through activation of Notch targets. Downstream of Notch, the transcription factor Kruppel-like factor 4 (Klf4) has been shown to 
be required for terminal differentiation of goblet cells (53). Expression of klf4a was shown to be controlled by Notch signaling (i.e., embryos treated with the $\gamma$-secretase inhibitor DAPT display increased klf4a expression in the intestine, while decreased klf4a expression and reduction in goblet cell number were observed in embryos injected with Notch intracellular domain mRNA; ref. 54). On the other hand, it has been demonstrated that the RA/RARa axis negatively modulates klf4 expression, thereby impacting goblet cell differentiation as well. In the future it will be interesting to investigate whether ApoB is involved in the interplay between the Notch and RA/ RARa pathways in the developing intestine.

Finally, the phenotypes detected in the liver and intestine of $a p o B$ mutants could result from the absence of fat-soluble vitamins, such as vitamins $\mathrm{E}$ and $\mathrm{A}$, and their RA metabolites, whose absorption and transport throughout the body rely mostly on $A p o B$, in the form of chylomicrons $(55,56)$.

Previous studies have demonstrated the strong impact of lipoproteins on EC behavior $(15,17,57,58)$; yet, the mechanisms by which $A p o B$ affects these cells are not fully understood. The double apoB mutant exhibited a marked angiogenic phenotype characterized by excessive sprouts in the SIVs, PCeV, and arterial intersomitic vessels. This angiogenic effect may result from a combination of factors related to ApoB deficiency, such as lipid raft density, lack of fat-soluble vitamins, and activation of downstream signaling pathways in ECs. Interestingly, the angiogenic defects were observed only following complete depletion of circulating ApoB lipoproteins. Although apoBb.1 mutants featured severe hypolipidemia, they did not display a strong angiogenic phenotype, suggesting that small amounts of ApoB are sufficient to maintain healthy control over the angiogenic process and further confirming the specific effects of the ApoB protein on EC behavior.

Our structure-function analyses revealed that APOB34, a truncated form of human APOB lacking the LDL receptor binding domain, is able to rescue the angiogenic phenotype in double $a p o B$ mutants. These results raise the hypothesis that an LDL receptor-independent pathway might regulate the effects of ApoB on ECs. Recently, it has been shown that LDL can bind the TGF- $\beta$ receptor activin receptor-like kinase 1 (ALK1; ref. 59), which is also involved in lipoproteins' transcytosis (60). Moreover, ALK1 has been shown to exert antiangiogenic effects via upregulation of VEGFR1 (61). Interestingly, stl mutants display reduced expression of vegfr1, leading to excessive angiogenesis (15). Therefore, it seems feasible that APOB induction of ALK1 activity leads to upregulation of vegfr1 and inhibition of angiogenesis.

Overall, our newly generated $a p o B$ mutants can serve as a model for studying human pathologies associated with hypolipidemia, as well as for related drug screens. Moreover, understanding the molecular mechanisms by which ApoB regulates angiogenesis will provide potential new therapeutic targets for the treatment of vascular pathologies ranging from cancer to ischemic heart disease.

\section{Methods}

Zebrafish husbandry and transgenic lines. Tg(fli1:EGFP)" stl (15), TgBAC(cldn15la-GFP)d1034 (34), Tg(fli1:dsRed $)^{u m 13}$ (62), $\operatorname{Tg}\left(\text { lyve1:dsRed2) }{ }^{\text {rz101 }} \text { (63), Tg(flt1_9a_cFos:GFP) }{ }^{w z 2} \text { (63), Tg(EPV.Tp1-Mmu.Hbb:EGFP) }\right)^{i a 12}$ (27), and Tg(-2.8fabp10a:EGFP) $)^{a s}$ (22) have been previously described.

To generate apo14:APOB25-IRESmCherry and apo14:APOB34-IRESmCherry, the zebrafish apo14 promoter (47) was cloned into pDONRP4-P1R (Invitrogen, Thermo Fisher Scientific), human APOB25 and APOB34 (45) were cloned into pDONR221 (Invitrogen, Thermo Fisher Scientific), and the IRESmCherry sequence was cloned into pDONRP2R-P3 (Invitrogen, Thermo Fisher Scientific), using Gateway BP Clonase II (Invitrogen, Thermo Fisher Scientific, 11789-020). The 3 vectors were then transferred into pDestTol2pA2 (62) using a Gateway LR reaction (Invitrogen, Thermo Fisher Scientific, 12538-120). The plasmids were injected along with Tol2 transposase mRNA into 1-cell-stage embryos (62).

Generation of zebrafish mutants. The apoBb.1 CRISPR guide was designed with CHOPCHOP; potential off-target sequences were assessed using the MIT CRISPR Design site. Oligonucleotides synthesized for the guide sequence GGACTAGTGTGGTCTTTGAC were cloned into the BsmBI sites of the pT7-gRNA plasmid (Addgene plasmid 46759). Cas 9 mRNA was generated from pCS2-nCas9n (64) using mMACHINE T7 ULTRA kit (Ambion, AM1345). Cas 9 mRNA (250 ng/ $\mu \mathrm{L})$ and guide RNAs (100 ng/ $\mu \mathrm{L})$ were coinjected into 1-cell-stage $\mathrm{Tg}(f l i 1: E G F P)^{{ }^{1}}$ transgenic embryos. For genotyping, genomic DNA was extracted at 24 hours postfertilization (hpf), amplified with 5'-TACTCTGTGAATGCCAGACAGG and 5'-TCAGAAGCTATCCAGCAAACA $(184 \mathrm{bp})$ primers, and analyzed on a $3 \%$ agarose gel. apoBa TALENs left - TACAGCTAACCTCAAGAA - and right - TGCCAGgtacaaaaaca plasmids were generated as described (65) and transcribed using mMESSAGE mMACHINE T7 ULTRA kit (Ambion, AM1345). mRNAs (250 ng each) were coinjected into 1-cell stage. For genotyping, genomic DNA was extracted at 24 
hpf, amplified with 5'-TATCAGTACACAGCAGAGAGCA and 5'-TCGTAAAATTAGGCTAAGCCA (110 bp), and analyzed on a 3\% agarose gel.

Antisense morpholino injection. The following antisense MOs (Gene Tools) were resuspended and injected as described (66) at the following concentrations: $m t p(15)(4 \mathrm{ng}), a p o B a(15)(4 \mathrm{ng})$, and apoBb.1 (CCATGATGGGTTCAGGTAAGCTCGT) (2 ng).

In situ hybridization and staining procedures. Embryos were fixed overnight in 4\% paraformaldehyde (PFA) and processed for ORO (15), AP (15), Nile red (MilliporeSigma, N3013; refs. 31, 32), and Alcian blue (MilliporeSigma, A3157; ref. 67) staining, as described. TUNEL staining was performed using In Situ Cell Death Detection Kit, TMR red (Roche, 12156792910), following manufacturer's instructions.

In situ hybridization was performed as described (68) using the following probes: fabp 10a (69) and prox $1 a$ (70). The following primers were used to generate the corresponding riboprobes: apoBa 5'-GACCTTGGCTTTCCGTTCC-3'， 5'-CAGCAGGGAAGCTCTCTATGAA-3'; apoBb.1 5'-GCTGCAGTGTATGCCATGGGAAT-3'， 5'-ATCAACAGTGGGTTCCAGACCCTT-3'; foxa2 5'-GTGTTACACCTCGGTCAGCA-3', 5'-CACTTGAAGCGCTTTTGCCT-3'; fabp2 5'-TGGAAAGTCGACCGCAATGAGA-3', 5'-TACCTTTCCGTTGTCCTTGCGT-3'; tfa 5'-TCTGGAGGCTGGAATACTCCTA-3', 5'-TAAACCTGAGCCCTTACGCA-3'.

Assessment of vascular phenotypes. Larvae were assayed for SIV ectopic sprouting using fluorescence imaging (PCeV, trunk) or AP staining (SIVs). Quantitation of the SIVs' phenotype was done using a grid lens in a Leica stereoscope under $\times 10$ original magnification; each side of the animal was scored separately.

Total RNA isolation and semiquantitative reverse transcription PCR. A pool of 10-20 embryos/sample was homogenized in TRIzol (Invitrogen, Thermo Fisher Scientific, 15596026) and processed for RNA extraction following standard procedures (15). A total of $1 \mu \mathrm{g}$ of RNA per reaction was reverse-transcribed using a High-Capacity cDNA Reverse Transcription Kit (Applied Biosystems, Thermo Fisher Scientific, 4368814). To measure relative changes in mRNA transcripts, we used the following primers: apoBa $5^{\prime}$-GACCTTGGCTTTCCGTTCC-3', 5'-CAGCAGGGAAGCTCTCTATGAA-3'; apoBb.1 5'-GCTGCAGTGTATGCCATGGGAAT-3', 5'-ATCAACAGTGGGTTCCAGACCCTT-3'; apoBb.2 5'-GTTCATAGGAGCGAGCATTGACCA-3', 5'-AGACCCAAACTGTCAACGAAAGGC-3'. Expression levels were standardized to the primer set specific for $\beta$-actin 5'-TGACAGGATGCAGAAGGAGA-3' and 5'-GCCTCCGATCCAGACAGAGT-3'.

$T G$ and cholesterol measurements. Twenty deyolked embryos at $3 \mathrm{dpf}$ per sample were gently homogenized in PBS with a pestle. After centrifugation at 15,000g for 15 minutes, supernatants were collected, and TG and cholesterol levels were measured using kits from BioVision (Triglyceride Quantification Kit, K622-100; Cholesterol Quantification Kit, K623-100) as described (71). For each genotype, 3 repeats of 10-20 embryos each were performed.

Protein extraction and Western blot. Zebrafish embryos at $3 \mathrm{dpf}$ were processed for Western blot as described (70). Briefly, proteins were separated by SDS-PAGE using a $6 \%$ separating gel and a $4 \%$ stacking gel. Transfer was performed at $400 \mathrm{~mA}$ for 1 hour. Membranes were blocked with 2\% BSA in PBS-Tween. The following antibodies were used: rabbit anti-human APOB100 (Abcam, ab20737), 1:1500; mouse monoclonal anti-human $\alpha$-tubulin (MilliporeSigma, T5168), 1:3000.

$O A$ and LDL treatments. OA (MilliporeSigma, O7501) was dissolved in ethanol and added to the fish water with fatty acid-free BSA (MilliporeSigma, A8806), at 6 to $20 \mu \mathrm{g} / \mathrm{mL}$ concentration.

Dil-LDL (Invitrogen, Thermo Fisher Scientific, L3482) was injected intravascularly at $2 \mathrm{dpf}$, as described (15).

Imaging. Confocal imaging was performed using a Zeiss LSM 780 upright confocal microscope (Carl Zeiss) with a W-Plan Apochromat $\times 20$ objective, NA 1.0. Images were processed using ImageJ (NIH). Fluorescent proteins were excited sequentially with $488 \mathrm{~nm}$ and $563 \mathrm{~nm}$ single-photon lasers.

Cell transfection and culture. HEK293 cells (2) that were 50\% confluent were cotransfected with $5 \mu \mathrm{g}$ Huh7MTP (15) and $10 \mu \mathrm{g}$ pGFP or APOB34-FLAG or APOB25-DsRed (45), using JET PEI (Polyplus, catalog 15021C1T) standard protocol. Twenty-four hours after transfection, medium was replaced with EBM-2 (endothelial basal medium) (Lonza, CC-3156), containing 0.5\% lipoprotein-depleted serum (2) and 1\% penicillin-streptomycin. Forty-eight hours later the medium was collected and centrifuged at $2000 \mathrm{rpm}$ for 5 minutes to ensure a cell-free medium. The upper phase was used as conditional medium for the tube formation assay.

Tube formation assay. HUVECs (Lonza) were cultured on gelatin-coated dishes, in M199 medium supplemented with 20\% FCS, $50 \mu \mathrm{g} / \mathrm{mL}$ Endothelial Cell Growth Supplement (Zotal catalog BT-203), $5 \mathrm{U} / \mathrm{mL}$ heparin, $1 \%$ penicillin-streptomycin, $2 \mathrm{mM}$ L-glutamine (16). 
For treatment, conditional medium collected from $M T P+G F P, M T P+G F P+\mathrm{LDL}(100 \mu \mathrm{g} / \mathrm{mL})(\mathrm{BT}-903)$, $M T P+A P O B 25$, and $M T P+A P O B 34$ transfected HEK293 cells was added. Following 18-hour incubation, 30,000 cells were seeded on Matrigel (BD, 356231) for 8 hours and cultured with the appropriate conditional medium $(M T P+\mathrm{GFP}, M T P+\mathrm{GFP}+\mathrm{LDL}, M T P+A P O B 25$, and $M T P+A P O B 34)$. Cells were then fixed in $4 \%$ PFA and imaged using a bright-field microscope. Nine fields/well were acquired per experiment. Total tube numbers were quantified using ImageJ. For each experiment, values were normalized to the nontreated well.

Statistics. All data are reported as mean values \pm SEM and were analyzed using Prism 5 software (GraphPad Software). Comparison of 2 samples was done by unpaired 2-tailed Student's $t$ test. Statistical significance for 3 or more samples was calculated via 1-way ANOVA followed by post hoc Tukey's for multiple comparisons. A $P$ value less than 0.05 was considered significant. In box-and-whisker plots, box indicates 25 th to 75 th percentiles, line indicates median, and whiskers represent min and max.

Study approval. Zebrafish were raised by standard methods and handled according to, and following approval by, the Weizmann Institute Animal Care and Use Committee (68).

\section{Author contributions}

HT designed and conducted all experiments, analyzed data, and cowrote the manuscript; IAD conducted HUVEC experiments; NM managed fish work and conducted zebrafish experiments; and KY initiated and directed the study, designed experiments, analyzed data, and cowrote the paper with input from all authors.

\section{Acknowledgments}

The authors would like to thank Yona Eli, Hila Raviv, and Lital Shen (Weizmann Institute) for technical assistance; S. Ben-Dor (Weizmann Institute) for bioinformatic analysis; Noa Stettner, Alon Harmelin, Gabriella Almog, Roy Hofi, and Anna Tatarin for superb animal care; Didier Stainier (Max Planck Institute for Heart and Lung Research) for providing Tg(-2.8fabp10a:EGFP) ${ }^{a s 3}$ fish; Michel Bagnat (Duke University Medical Center) for providing the $\operatorname{TgBAC}\left(\mathrm{cldn} 15 \mathrm{la}-\right.$ GFP) ${ }^{\text {pdlo34 }}$ transgenic line; and Gregory S. Shelness (NIH) for the APO25and APOB34 plasmids. The authors are grateful to all the members of the Yaniv lab for discussion, technical assistance, and continuous support. This work was supported in part by European Research Council 335605 (to KY); Israel Science Foundation 861/2013 (to KY); the H\&M Kimmel Institute for Stem Cell Research; the estate of Emile Mimran (SABRA program); the Willner Family Center for Vascular Biology; the estate of Paul Ourieff; the Carolito Stiftung; Lois Rosen, Los Angeles, California, USA; Edith Frumin; the Fondazione Henry Krenter; the Wallach Hanna \& Georges Lustgarten Fund; the Polen Charitable Trust; and the Daniel Shapiro Cardiovascular Fund. KY is the incumbent of the Enid Barden and Aaron J. Jade Professorial Chair in Memory of Canter John Y. Jade.

Address correspondence to: Karina Yaniv, Department of Biological Regulation, Weizmann Institute of Science, 234 Herzl St., Rehovot 76100, Israel. Phone: 972.8.934.2224; Email: karina.yaniv@weizmann.ac.i1.

1. Olofsson S-O, Borèn J. Apolipoprotein B: a clinically important apolipoprotein which assembles atherogenic lipoproteins and promotes the development of atherosclerosis. J Intern Med. 2005;258(5):395-410.

2. Avraham-Davidi I, et al. Lipid signaling in the endothelium. Exp Cell Res. 2013;319(9):1298-1305.

3. Davidson NO, Shelness GS. Apolipoprotein B: mRNA editing, lipoprotein assembly, and presecretory degradation. Annu Rev Nutr. 2000;20:169-193.

4. Welty FK. Hypobetalipoproteinemia and abetalipoproteinemia. Curr Opin Lipidol. 2014;25(3):161-168.

5. Schonfeld G, et al. Familial hypobetalipoproteinemia: genetics and metabolism. Cell Mol Life Sci. 2005;62(12):1372-1378.

6. Nass KJ, et al. High prevalence of apolipoprotein B dyslipoproteinemias in non-alcoholic fatty liver disease: the lifelines cohort study. Metabolism. 2017;72:37-46.

7. Chatrath $\mathrm{H}$, et al. Dyslipidemia in patients with nonalcoholic fatty liver disease. Semin Liver Dis. 2012;32(1):22-29.

8. Katsiki N, et al. Non-alcoholic fatty liver disease and dyslipidemia: an update. Metabolism. 2016;65(8):1109-1123.

9. Di Filippo M, et al. Homozygous MTTP and APOB mutations may lead to hepatic steatosis and fibrosis despite metabolic differences in congenital hypocholesterolemia. J Hepatol. 2014;61(4):891-902.

10. Tanoli T, et al. Fatty liver in familial hypobetalipoproteinemia: roles of the APOB defects, intra-abdominal adipose tissue, and insulin sensitivity. J Lipid Res. 2004;45(5):941-947.

11. von Scheidt M, et al. Applications and limitations of mouse models for understanding human atherosclerosis. Cell Metab. $2017 ; 25(2): 248-261$

12. Kim E, Young SG. Genetically modified mice for the study of apolipoprotein B. J Lipid Res. 1998;39(4):703-723.

13. Anderson JL, et al. Zebrafish lipid metabolism: from mediating early patterning to the metabolism of dietary fat and cholesterol. Methods Cell Biol. 2011;101:111-141. 
14. Otis JP, et al. Zebrafish as a model for apolipoprotein biology: comprehensive expression analysis and a role for ApoA-IV in regulating food intake. Dis Model Mech. 2015;8(3):295-309.

15. Avraham-Davidi I, et al. ApoB-containing lipoproteins regulate angiogenesis by modulating expression of VEGF receptor 1. Nat Med. 2012;18(6):967-973.

16. Gibbs-Bar L, et al. Autotaxin-lysophosphatidic acid axis acts downstream of apoprotein B lipoproteins in endothelial cells. Arterioscler Thromb Vasc Biol. 2016;36(10):2058-2067.

17. Stoletov K, et al. Vascular lipid accumulation, lipoprotein oxidation, and macrophage lipid uptake in hypercholesterolemic zebrafish. Circ Res. 2009;104(8):952-960.

18. Ka J, Jin SW. Zebrafish as an emerging model for dyslipidemia and associated diseases. J Lipid Atheroscler. 2021;10(1):42-56.

19. Thierer JH, et al. The LipoGlo reporter system for sensitive and specific monitoring of atherogenic lipoproteins. Nat Commun. 2019;10(1):3426.

20. Isogai S, et al. Angiogenic network formation in the developing vertebrate trunk. Development. 2003;130(21):5281-5290.

21. Wilson $\mathrm{MH}$, et al. A point mutation decouples the lipid transfer activities of microsomal triglyceride transfer protein. PLoS Genet. 2020;16(8):e1008941.

22. Her GM, et al. In vivo studies of liver-type fatty acid binding protein (L-FABP) gene expression in liver of transgenic zebrafish (Danio rerio). FEBS Lett. 2003;538(1-3):125-133.

23. Seth A, et al. Prox 1 ablation in hepatic progenitors causes defective hepatocyte specification and increases biliary cell commitment Development. 2014;141(3):538-547.

24. Field HA, et al. Formation of the digestive system in zebrafish. I. Liver morphogenesis. Dev Biol. 2003;253(2):279-290.

25. Mudumana SP, et al. Expression analyses of zebrafish transferrin, ifabp, and elastaseB mRNAs as differentiation markers for the three major endodermal organs: liver, intestine, and exocrine pancreas. Dev Dyn. 2004;230(1):165-173.

26. Tao T, Peng J. Liver development in zebrafish (Danio rerio). J Genet Genomics. 2009;36(6):325-334.

27. Hen G, et al. Venous-derived angioblasts generate organ-specific vessels during zebrafish embryonic development. Development 2015;142(24):4266-4278.

28. Lorent $\mathrm{K}$, et al. Inhibition of Jagged-mediated Notch signaling disrupts zebrafish biliary development and generates multi-organ defects compatible with an Alagille syndrome phenocopy. Development. 2004;131(22):5753-5766.

29. de Bruin TWA, et al. Radiological evidence of nonalcoholic fatty liver disease in familial combined hyperlipidemia. Am $J$ Med. 2004;116(12):847-849.

30. Kneeman JM, et al. Secondary causes of nonalcoholic fatty liver disease. Therap Adv Gastroenterol. 2012;5(3):199-207.

31. Greenspan P, et al. Nile red: a selective fluorescent stain for intracellular lipid droplets. J Cell Biol. 1985;100(3):965-973.

32. Tingaud-Sequeira A, et al. Zebrafish obesogenic test: a tool for screening molecules that target adiposity. J Lipid Res. 2011;52(9):1765-1772.

33. Wallace KN, Pack M. Unique and conserved aspects of gut development in zebrafish. Dev Biol. 2003;255(1):12-29.

34. Alvers AL, et al. Single continuous lumen formation in the zebrafish gut is mediated by smoothened-dependent tissue remodeling. Development. 2014;141(5):1110-1119.

35. Pack M, et al. Mutations affecting development of zebrafish digestive organs. Development. 1996;123(1):321-328

36. Xie Y, et al. Compensatory increase in hepatic lipogenesis in mice with conditional intestine-specific Mttp deficiency. J Biol Chem. 2006;281(7):4075-4086.

37. Young SG, et al. A genetic model for absent chylomicron formation: mice producing apolipoprotein B in the liver, but not in the intestine. J Clin Invest. 1995;96(6):2932-2946.

38. Raabe M, et al. Knockout of the abetalipoproteinemia gene in mice: reduced lipoprotein secretion in heterozygotes and embryonic lethality in homozygotes. Proc Natl Acad Sci U S A. 1998;95(15):8686-8691.

39. Oyarbide U, et al. Loss of Sbds in zebrafish leads to neutropenia and pancreas and liver atrophy. JCI Insight. 2020;5(17):e134309.

40. Crosnier C, et al. Delta-Notch signalling controls commitment to a secretory fate in the zebrafish intestine. Development. 2005;132(5):1093-1104

41. Fang L, et al. Zebrafish models of dyslipidemia: relevance to atherosclerosis and angiogenesis. Transl Res. 2014;163(2):99-108

42. Goi M, Childs SJ. Patterning mechanisms of the sub-intestinal venous plexus in zebrafish. Dev Biol. 2016;409(1):114-128.

43. Chappell JC, et al. Flt-1 (VEGFR-1) is essential for the VEGF-Notch feedback loop during angiogenesis. Arterioscler Thromb Vasc Biol. 2013;33(8):1952-1959.

44. Chappell JC, et al. Flt-1 (VEGFR-1) coordinates discrete stages of blood vessel formation. Cardiovasc Res. 2016;111(1):84-93.

45. Shelness GS, et al. Identification of the lipoprotein initiating domain of apolipoprotein B. J Biol Chem. 2003;278(45):44702-44707.

46. Ingram MF, Shelness GS. Folding of the amino-terminal domain of apolipoprotein B initiates microsomal triglyceride transfer protein-dependent lipid transfer to nascent very low density lipoprotein. J Biol Chem. 1997;272(15):10279-10286.

47. Wang R, et al. An Apo-14 promoter-driven transgenic zebrafish that marks liver organogenesis. PLoS One. 2011;6(7):e22555

48. Linton M, et al. Familial hypobetalipoproteinemia. J Lipid Res. 1993;34(4):521-541.

49. Garnaas MK, et al. Rargb regulates organ laterality in a zebrafish model of right atrial isomerism. Dev Biol. 2012;372(2):178-189.

50. Li Y, et al. The multifaceted nature of retinoid transport and metabolism. Hepatobiliary Surg Nutr. 2014;3(3):126-139.

51. Yee NS, et al. Mutation of RNA Pol III subunit rpc2/polr3b leads to deficiency of Subunit Rpc11 and disrupts zebrafish digestive development. PLoS Biol. 2007;5(11):e312.

52. Jijon HB, et al. Intestinal epithelial cell-specific RAR $\alpha$ depletion results in aberrant epithelial cell homeostasis and underdeveloped immune system. Mucosal Immunol. 2018;11(3):703-715.

53. Katz JP, et al. The zinc-finger transcription factor Klf4 is required for terminal differentiation of goblet cells in the colon. Development. 2002;129(11):2619-2628.

54. Li I-C, et al. Zebrafish Krüppel-like factor 4a represses intestinal cell proliferation and promotes differentiation of intestinal cell lineages. PLoS One. 2011;6(6):e20974.

55. Niederreither K, Dollé P. Retinoic acid in development: towards an integrated view. Nat Rev Genet. 2008;9(7):541-553

56. Oehlers SH, et al. Retinoic acid suppresses intestinal mucus production and exacerbates experimental enterocolitis. Dis Model Mech. 2012;5(4):457-467. 
57. Zabroski IO, Nugent MA. Lipid raft association stabilizes VEGF Receptor 2 in endothelial cells. Int J Mol Sci. 2021;22(2):798. 58. Fang L, Miller YI. Regulation of lipid rafts, angiogenesis and inflammation by AIBP. Curr Opin Lipidol. 2019;30(3):218-223.

59. Kraehling JR, et al. Genome-wide RNAi screen reveals ALK1 mediates LDL uptake and transcytosis in endothelial cells. Nat Commun. 2016;7:13516

60. Zhang X, et al. Endothelial transcytosis of lipoproteins in atherosclerosis. Front Cardiovasc Med. 2018;5:130.

61. Larrivée B, et al. ALK1 signaling inhibits angiogenesis by cooperating with the Notch pathway. Dev Cell. 2012;22(3):489-500.

62. Villefranc JA, et al. Gateway compatible vectors for analysis of gene function in the zebrafish. Dev Dyn. 2007;236(11):3077-3087.

63. Nicenboim J, et al. Lymphatic vessels arise from specialized angioblasts within a venous niche. Nature. 2015;522(7554):56-61.

64. Jao LE, et al. Efficient multiplex biallelic zebrafish genome editing using a CRISPR nuclease system. Proc Natl Acad Sci U S A. 2013;110(34):13904-13909.

65. Reyon D, et al. Engineering designer transcription activator-like effector nucleases (TALENs) by REAL or REAL-Fast assembly. Curr Protoc Mol Biol. 2012; Chapter 12:Unit 12.15.

66. Cohen B, et al. BACH family members regulate angiogenesis and lymphangiogenesis by modulating VEGFC expression. Life Sci Alliance. 2020;3(4):e202000666.

67. Chen YH, et al. Zebrafish cdx1b regulates differentiation of various intestinal cell lineages. Dev Dyn. 2009;238(5):1021-1032

68. Jerafi-Vider A, et al. VEGFC induced cell cycle arrest mediates sprouting and differentiation of venous and lymphatic endothelial cells [preprint]. https://doi.org/10.1101/2020.06.17.155028. Posted on bioRxiv June 19, 2020.

69. Stuckenholz C, et al. Sfrp5 modulates both Wnt and BMP signaling and regulates gastrointestinal organogensis in the zebrafish, danio rerio. PLoS One. 2013;8(4):e62470.

70. Yaniv K, et al. Live imaging of lymphatic development in the zebrafish. Nat Med. 2006;12(6):711-716.

71. Liu C, et al. Apoc2 loss-of-function zebrafish mutant as a genetic model of hyperlipidemia. Dis Model Mech. 2015;8(8):989-998. 